\title{
A COUNTEREXAMPLE TO THE NODAL DOMAIN CONJECTURE AND A RELATED SEMILINEAR EQUATION
}

\author{
CHANG-SHOU LIN AND WEI-MING NI \\ (Communicated by Walter Littman)
}

\begin{abstract}
In this paper we first establish a nonuniqueness result for a semilinear Dirichlet problem of which the nonlinearity is of super-critical growth. We then apply this result to construct a Schrödinger operator on a domain $\Omega$ such that the second eigenfunctions of this operator (with zero Dirichlet boundary data) have their nodal sets completely contained in the interior of the domain $\Omega$.
\end{abstract}

1. Introduction. In this paper we shall consider the eigenvalue problem

$$
\begin{cases}(\Delta+V) \varphi+\lambda \varphi=0 & \text { in } \Omega, \\ \varphi=0 & \text { on } \partial \Omega,\end{cases}
$$

where $\Delta=\sum_{i=1}^{n}\left(\partial^{2} / \partial x_{i}^{2}\right)$ is the Laplace operator, $V$ is a given smooth function (the "potential"), $\lambda$ is an eigenvalue and $\Omega$ is a bounded smooth domain in $\mathbf{R}^{n}$, $n \geq 2$. The set $\{x \in \Omega \mid \varphi(x)=0\}$ is called the nodal set of $\varphi$. It is well known that the first eigenfunction is always positive in $\Omega$ while all the higher eigenfunctions must change sign. The only general theorem concerning higher eigenfunctions seems to be the Courant Nodal Domain Theorem [2] which asserts that the nodal set of a kth eigenfunction divides the domain $\Omega$ into at most $k$ subregions. In general the topology of nodal domains is not known, even in the simplest case $n=2, k=2$ and $V \equiv 0$. In case $\Omega \subseteq \mathbf{R}^{2}$ is convex and $V \equiv 0$ in $\Omega$, the following conjecture has been around for quite some time (see e.g. [8]).

NODAL DOMAIN CONJECTURE. The nodal line of any second eigenfunction must intersect the boundary $\partial \Omega$ at exactly two points.

Progress has been made by L. Payne in [7] where this conjecture is verified under the additional hypothesis that the domain $\Omega$ is symmetric with respect to a line. Recently, in [4], the first author has established this conjecture for bounded convex domains $\Omega$ which are symmetric with respect to a point or are invariant under a rotation of a fixed (but arbitrary) angle.

The purpose of this paper is to show that the above conjecture is false when $V \not \equiv 0$. In fact, we shall construct, for each dimension $n \geq 2$, a potential $V$ which is radially symmetric while the domain $\Omega$ is a ball and yet a second eigenfunction $\varphi_{2}$ of (1.1) is also radially symmetric. This, in turn, implies that the nodal set of $\varphi_{2}$ is a sphere and thus encloses a smaller ball in $\Omega$.

Received by the editors September 26, 1986.

1980 Mathematics Subject Classification (1985 Revision). Primary 35J60, 35P99.

Research supported in part by the National Science Foundation. 
To achieve this purpose, in case $n \geq 3$, we shall study the semilinear elliptic equation

$$
\Delta u+u^{p}+u^{q}=0
$$

where

$$
p=\frac{q+1}{2} \text { and } \frac{n+2}{n-2}<q<\frac{n+6}{n-2} .
$$

We first show that equation (1.2) possesses a positive entire radial solution in $\mathbf{R}^{n}$. It then follows that there exists $R_{*}>0$ such that for every $R>R_{*}$, equation (1.2) has at least two positive radial solutions in $B_{R}$ (i.e. the ball with radius $R$ ) with zero Dirichlet boundary data. The existence of positive entire solutions and the nonuniqueness property of equation (1.2) also seem to be new in $\mathbf{R}^{n}, n \geq 3$, and are of independent interest. A similar nonuniqueness result in $\mathbf{R}^{\mathbf{2}}$ was found earlier by $\mathrm{Ni}$ and Nussbaum [5]. Using these results, we are then able to construct (in $\S 3$ below) the radial potential $V$ described above.

ACKNOWLEDGMENT. This paper was completed while both authors were visiting the Centre for Mathematical Analysis, Australian National University. The authors wish to thank the Centre for the invitation and the hospitality during their stay in Canberra.

2. A semilinear equation. In this section we shall study positive radial solutions of the semilinear equation

$$
\Delta u+f(u)=0
$$

in $\mathbf{R}^{n}$ as well as in $B_{R}$ (ball of radius $R$ ) $\subseteq \mathbf{R}^{n}, n \geq 3$, where

$$
f(u)= \begin{cases}u^{p}+u^{q}, & u \geq 0 \\ 0, & u<0\end{cases}
$$

with

$$
1<p<(n+2) /(n-2)<q .
$$

Since we are only interested in radial solutions, equation (2.1) reduces to an ordinary differential equation. We consider the corresponding initial-value problem

$$
\left\{\begin{array}{l}
u_{r r}+(n-1) u_{r} / r+f(u)=0 \\
u(0)=\alpha>0 \\
u_{r}(0)=0
\end{array}\right.
$$

and denote the solution by $u=u(r ; \alpha)$.

PROPOSITION 2.4. There exists a $\delta>0$ such that for all $\alpha \in(0, \delta)$ the solution $u(r ; \alpha)$ must vanish for some finite $r$.

PROOF. For each $\alpha>0$, set $w(x)=u(r ; \alpha) / \alpha, s=\alpha^{(p-1) / 2} r$ and $\varepsilon=\alpha^{q-p}$. Then $w$ satisfies

$$
\left\{\begin{array}{l}
w_{s s}+(n-1) w_{s} / s+f(w, \varepsilon)=0 \\
w(0)=1 \\
w_{s}(0)=0
\end{array}\right.
$$


where

$$
f(w, \varepsilon)= \begin{cases}w^{p}+\varepsilon w^{q} & \text { if } w \geq 0 \\ 0, & \text { if } w<0\end{cases}
$$

Observe that equation (2.5) makes sense for all $\varepsilon \in \mathbf{R}$. For each $\varepsilon \in \mathbf{R}$, we denote the solution $w$ of $(2.5)$ by $w(s, \varepsilon)$. It is well known that $w(s, 0)$ has exactly one zero and $w(s, 0)$ remains negative after its zero since, by the maximum principle, $w(s, \varepsilon)$ is always decreasing for every $\varepsilon \geq 0$. Now the continuous dependence of $w(s, \varepsilon)$ on $\varepsilon$ implies that there exists an $\varepsilon_{0}>0$ such that $w(s, \varepsilon)$ has a zero for every $0<\varepsilon \leq \varepsilon_{0}$. (Note that since $w(s, \varepsilon)$ is decreasing for all $\varepsilon>0$, the term $\varepsilon w^{q}$ is bounded by $\varepsilon w^{q}(0)=\varepsilon$ when $w(s)$ is positive.) Set $\delta=\varepsilon_{0}^{1 /(q-p)}$, the result follows. Q.E.D.

In the rest of this paper, we shall denote the first zero $u(r ; \alpha)$ by $\rho(\alpha)$ with the convention that $\rho(\alpha)=\infty$ if $u(r ; \alpha)$ is a positive entire solution. Again, note that $u(r ; \alpha)$ is always decreasing and has at most one zero.

PROPOSITION 2.6. $\rho(\alpha) \rightarrow+\infty$ as $\alpha \rightarrow 0$.

PROOF. Multiplying (2.1) by $u$ and integrating over $B_{\rho(\alpha)}$, we obtain

$$
\begin{aligned}
\int_{B_{\rho(\alpha)}}|D u|^{2} d x & =\int_{B_{\rho(\alpha)}}\left(u^{p+1}+u^{q+1}\right) \\
& \leq\left(\alpha^{p-1}+\alpha^{q-1}\right) \int_{B_{\rho(\alpha)}} u^{2}
\end{aligned}
$$

since $u(r ; \alpha) \leq u(0 ; \alpha)=\alpha$. By Poincaré's inequality, there exists a constant $C_{0}>0$, independent of $\alpha$, such that

$$
\frac{C_{0}}{\rho^{2}(\alpha)} \int_{B_{\rho(\alpha)}} u^{2} \leq \int_{B_{\rho(\alpha)}}|D u|^{2}
$$

Therefore,

$$
\rho^{2}(\alpha) \geq \frac{C_{0}}{\alpha^{p-1}+\alpha^{q-1}}
$$

and our conclusion follows. Q.E.D.

We now exhibit entire solutions of (2.1). Let $p=(q+1) / 2$. Condition (2.2) implies that

$$
\frac{n+2}{n-2}<q<\frac{n+6}{n-2}
$$

Set

$$
\tilde{u}(r)=\frac{1}{\zeta}\left(\frac{\xi^{2}}{\xi^{2}+r^{2}}\right)^{2 /(q-1)}
$$

where

$$
\varsigma=\left[\frac{n-2}{2(q+1)}\left(q-\frac{n+2}{n-2}\right)\right]^{2 /(q-1)}, \quad \xi=\frac{n-2}{q-1}\left(q-\frac{n+2}{n-2}\right)\left(\frac{2}{q+1}\right)^{1 / 2} .
$$

Straightforward computation leads to the following proposition. 
PROPOSITION 2.8. $\tilde{u}$ is a positive radial entire solution of (2.1).

Now set $A=\{\alpha>0 \mid \rho(\alpha)<\infty\}$. It is clear that $A$ is an open set. By Propositions 2.4 and 2.8, we see that there exists an interval $(0, \beta) \subseteq A$ with $\beta \notin A$. By Proposition 2.6 and the continuous dependence on initial values, we have

$$
\lim _{\alpha \downarrow 0} \rho(\alpha)=\infty=\lim _{\alpha \uparrow \beta} \rho(\alpha) .
$$

Letting $R_{*}=\inf \{\rho(\alpha) \mid \alpha \in(0, \beta)\}$, we see that there exists an $\alpha_{*} \in(0, \beta)$ such that $\rho\left(\alpha_{*}\right)=R_{*}$. Moreover, (2.9) also gives the following

THEOREM 2.10. Suppose that (2.7) holds. Then for every $R>R_{*}$ the Dirichlet problem

$$
\begin{cases}\Delta u+u^{(q+1) / 2}+u^{q}=0, & \text { in } B_{R}, \\ u=0, & \text { on } \partial B_{R},\end{cases}
$$

possesses at least two positive radial solutions.

3. A counterexample. The main goal of this section is to construct a potential $V$ for which the Nodal Domain Conjecture (stated in $\S 1$ ) is false. In particular, the nodal set of a second eigenfunction of $\Delta+V$ (of (1.1)) does not intersect the boundary $\partial \Omega$ and it encloses a region inside $\Omega$.

We start with a general observation. Let $u$ be a positive solution of

$$
\begin{cases}\Delta u+g(u)=0, & \text { in } B_{\rho}, \\ u=0, & \text { on } \partial B_{\rho},\end{cases}
$$

where $g$ is a $C^{1}$-function, and let $\varphi$ be an eigenfunction of the linearized equation at $u$; i.e.

$$
\begin{cases}\Delta \varphi+g^{\prime}(u) \varphi+\lambda \varphi=0, & \text { in } B_{\rho}, \\ \varphi=0, & \text { on } \partial B_{\rho} .\end{cases}
$$

PROPOSITION 3.3. If $\lambda \leq 0$, then $\varphi$ must be radially symmetric.

ProOF. Let $0=\mu_{1}<(n-1)=\mu_{2} \leq \mu_{3} \leq \cdots$ be the eigenvalues of the Laplacian on the standard unit sphere $S^{n-1}$, and $e_{k}(\theta), \theta \in S^{n-1}$, be the corresponding eigenfunctions, $k=1,2, \ldots$. Suppose that $\varphi$ satisfies equation (3.2). Set

$$
\varphi_{k}(r)=\int_{S^{n-1}} \varphi(r, \theta) e_{k}(\theta) d \theta
$$

For $k \geq 2, \varphi_{k}(0)=\varphi_{k}(\rho)=0$ and $\varphi_{k}$ satisfies

$$
\varphi_{k}^{\prime \prime}+\frac{n-1}{r} \varphi_{k}^{\prime}+\left[g^{\prime}(u(r))-\frac{\mu_{k}}{r^{2}}\right] \varphi_{k}+\lambda \varphi_{k}=0 .
$$

We claim that $\varphi_{k} \equiv 0$ for all $k \geq 2$. Suppose on the contrary that $\varphi_{k} \not \equiv 0$. Let $\rho_{0}$ be the first positive zero of $\varphi_{k}$; then we may assume without loss of generality that $\varphi_{k}>0$ in $\left(0, \rho_{0}\right)$. Since $u$ is radial and decreasing in $B_{\rho}[3]$, differentiating (3.1) with respect to $r$ gives

$$
\left\{\begin{array}{l}
\left(u^{\prime}\right)^{\prime \prime}+\frac{n-1}{r}\left(u^{\prime}\right)+\left(g^{\prime}(u(r))-\frac{n-1}{r^{2}}\right) u^{\prime}=0 \\
u^{\prime}(0)=0, \quad u^{\prime}<0 \text { in }\left(0, \rho_{0}\right] .
\end{array}\right.
$$


Multiplying (3.4) by $r^{n-1} u^{\prime}$ and integrating the resulting equation from 0 to $\rho_{0}$, we obtain, from (3.5) that

$$
\varphi_{k}^{\prime}\left(\rho_{0}\right) u^{\prime}\left(\rho_{0}\right) \rho_{0}^{n-1}+\int_{0}^{\rho_{0}} \frac{(n-1)-\mu_{k}}{r^{2}} \varphi_{k} u^{\prime} r^{n-1} d r=-\lambda \int_{0}^{\rho_{0}} \varphi_{k} u^{\prime} r^{n-1} d r .
$$

Since $\varphi_{k}^{\prime}\left(\rho_{0}\right)<0,(n-1) \leq \mu_{k}$, the left-hand side is positive. Thus $\lambda$ must be positive, a contradiction. Hence $\varphi_{k} \equiv 0$ for all $k \geq 2$ and therefore $\varphi$ is radially symmetric. Q.E.D.

We now come to the main result of this section.

THEOREM 3.6. There exists a radially symmetric potential $V$ and a ball $B_{R}$. such that all the second eigenfunctions of $\Delta+V$ in $B_{R_{*}}$ with zero Dirichlet boundary data are radially symmetric.

REMARKS. (i) The theorem above implies that the Nodal Domain Conjecture is false for $\Delta+V$ in general.

(ii) In the theorem above, there is only one second eigenfunction since all second eigenfunctions are radial.

ProOF OF THEOREM 3.6. First, we prove the case $n \geq 3$. We shall use the same notations as in $\S 2$. Let $u=u(r ; \alpha)$ be the solution of $(2.3)$ with exponents $p, q$ satisfying (1.3). Since $u(\rho(\alpha) ; \alpha)=0$ for all $\alpha$ in $(0, \beta)$ and $\rho(\alpha)$ assumes its minimum $R_{*}$ (for $\alpha \in(0, \beta)$ ) at $\alpha_{*}$, we have

$$
\frac{\partial u}{\partial \alpha}\left(\rho\left(\alpha_{*}\right) ; \alpha_{*}\right)=-\frac{\partial u}{\partial r}\left(\rho\left(\alpha_{*}\right) ; \alpha_{*}\right) \frac{d \rho}{d \alpha}\left(\alpha_{*}\right)=0
$$

Set $u_{*}(r)=u\left(r ; \alpha_{*}\right), \varphi(r)=\partial u\left(r ; \alpha_{*}\right) / \partial \alpha$. Then $\varphi$ satisfies

$$
\begin{cases}\Delta \varphi+\left(p u_{*}^{p-1}+q u_{*}^{q-1}\right) \varphi=0, & \text { in } B_{R_{*}} \\ \varphi=0, & \text { on } \partial B_{R_{*}}\end{cases}
$$

We claim that 0 is not the first eigenvalue of the potential $p u_{*}^{p-1}+q u_{*}^{q-1}$ in $B_{R_{*}}$. For, otherwise, we have $\varphi>0$ in $B_{R_{*}}$. Thus

$$
\begin{aligned}
0 & =\int_{B_{R_{*}}} u_{*}\left[\Delta \varphi+\left(p u_{*}^{p-1}+q u_{*}^{q-1}\right) \varphi\right] d x \\
& =\int_{B_{R_{*}}} \varphi\left[\Delta u_{*}+p u_{*}^{p}+q u_{*}^{q}\right] d x \\
& =\int_{B_{R_{*}}} \varphi\left[(p-1) u_{*}^{p}+(q-1) u_{*}^{q}\right] d x,
\end{aligned}
$$

but the last integral is positive, a contradiction. Setting

$$
V(r)=p u_{*}^{p-1}(r)+q u_{*}^{q-1}(r)
$$

in $B_{R_{*}}$, we see that the above assertion implies that the second eigenvalue of the potential $V$ in (3.7), $\lambda_{2}(V)$, must be less than or equal to zero. By Proposition 3.3, all the corresponding second eigenfunctions must be radial (thus there is one such eigenfunction), and $V$, defined by (3.7), is the desired potential. 
For the case $n=2$, we need a similar nonuniqueness result from [5]. In [5, Theorem 4.23, p. 91], Ni and Nussbaum constructed a function $\tilde{f}(u)$ satisfying

(i) $\tilde{f}(0)=\tilde{f}^{\prime}(0)=0, \tilde{f}(u)>0$ for $u>0$,

(ii) $\tilde{f}$ is smooth and convex,

(iii) the Dirichlet problem

$$
\begin{cases}\Delta u+\tilde{f}(u)=0, & \text { in } B_{b}, \\ u=0, & \text { on } \partial B_{b},\end{cases}
$$

possesses at least two positive radial solutions for some $b>0$. Suppose that these two positive solutions are $u\left(r ; \alpha_{1}\right)$ and $u\left(r ; \alpha_{2}\right), \alpha_{2}>\alpha_{1}$. It is well known that when $n=2, u(r ; \alpha)$ must have a finite zero for any $\alpha>0$ since $\tilde{f}(u)>0$ for $u>0$ (see e.g. Theorem 2.0 in [6]). Since $\rho\left(\alpha_{1}\right)=\rho\left(\alpha_{2}\right)=b$ (recall that $\rho(\alpha)$ is the first zero of $u(r ; \alpha))$, there exists a critical point $\alpha_{*} \in\left(\alpha_{1}, \alpha_{2}\right)$ such that $\rho^{\prime}\left(\alpha_{*}\right)=0$. As before, set $R_{*}=\rho\left(\alpha_{*}\right), u_{*}=u\left(r ; \alpha_{*}\right)$ and $\varphi(r)=\partial u\left(r ; \alpha_{*}\right) / \partial \alpha, \varphi(r)$ then satisfies

$$
\begin{cases}\Delta \varphi+\tilde{f}^{\prime}(u) \varphi=0, & \text { in } B_{R_{*}}, \\ \varphi=0, & \text { on } \partial B_{R_{*}} .\end{cases}
$$

By (i), (ii) and the above arguments for the case $n \geq 3$ it is easy to see that the second eigenvalue of the Schrödinger operator $\Delta+\tilde{f}^{\prime}\left(u_{*}\right)$ is less than or equal to zero. We now conclude as in the previous case that $V(r)=\tilde{f}^{\prime}\left(u_{*}(r)\right)$ is the desired potential. Q.E.D.

As a consequence of the above theorem, we have

THEOREM 3.8. There exists a radially symmetric potential $\tilde{V}$ and a ball $B_{R_{*}}$ such that the multiplicity of the second eigenvalue of $\Delta+\tilde{V}$ in $B_{R_{*}}$ with zero Dirichlet boundary data is $n+1$, and one of the corresponding second eigenfunctions is radially symmetric.

Proof. Let $V$ and $B_{R_{*}}$ be given by Theorem 3.6. Consider $\{t V \mid 0 \leq t \leq 1\}$. Let $\lambda_{2}(t)$ be the second eigenvalue of $\Delta+t V$ in (1.1); then $\lambda_{2}(t)$ is continuous in $t$ (see [2]). Note that $\lambda_{2}(0)$ has multiplicity $n$ and $\lambda_{2}(1)$ has multiplicity 1 with the corresponding eigenfunction being radial. Now let

$$
\begin{array}{r}
t_{0}=\inf \{t>0 \mid \text { at least one of the second eigenfunctions } \\
\text { corresponding to } \left.\lambda_{2}(t) \text { is radial }\right\} .
\end{array}
$$

We assert that $\lambda_{2}\left(t_{0}\right)$ has multiplicity $n+1$; in fact, we claim that $\lambda_{2}\left(t_{0}\right)$ has one radial eigenfunction and $n$ nonradial eigenfunctions.

By the definition of $t_{0}$, there exists a sequence $s_{1}>s_{2}>\cdots$ with $s_{j} \rightarrow t_{0}$ and for each $s_{j}$, there exists a radial second eigenfunction $\varphi_{j}$ with $\left\|\varphi_{j}\right\|_{L^{2}}=1$. From standard elliptic estimates it follows that (passing to a subsequence if necessary) $\varphi_{j}$ converges (in $C^{2}\left(\bar{B}_{R_{*}}\right)$ ) to a nonzero radial function $\varphi_{0}$. Since $\lambda_{2}\left(s_{j}\right) \rightarrow \lambda_{2}\left(t_{0}\right)$, $\varphi_{0}$ is a radial second eigenfunction of $\lambda_{2}\left(t_{0}\right)$. Thus $t_{0}$ is assumed and is therefore positive. It remains to show that there are $n$ nonradial second eigenfunctions corresponding to $\lambda_{2}\left(t_{0}\right)$. To achieve this, we need the following

LEMMA 3.9. If $\psi$ is a nonradial second eigenfunction of

$$
\begin{cases}(\Delta+W) \psi+\lambda \psi=0, & \text { in } B_{1}, \\ \psi=0, & \text { on } \partial B_{1},\end{cases}
$$


where the potential $W$ is a smooth radial function, then $\psi$ must take the form (up to a rotation) $\varphi(r) x_{i} / r$ for some $1 \leq i \leq n$, where $\varphi$ satisfies

$$
\varphi^{\prime \prime}+\frac{n-1}{r} \varphi^{\prime}+\left(W-\frac{n-1}{r^{2}}\right) \varphi+\lambda \varphi=0
$$

for $r \in(0,1)$ with $\varphi(0)=0=\varphi(1)$ and $\varphi>0$ in $(0,1)$.

Coming back to the proof of the theorem, we recall that $t_{0}>0$. Thus there is a sequence $t_{1} \leq t_{2} \leq \cdots$ with $t_{j} \rightarrow t_{0}$ as $j \rightarrow \infty$ such that for each $t_{j}$, there are $n$ nonradial second eigenfunctions of the form $\varphi_{j}(r) x_{i} / r, i=1, \ldots, n$, corresponding to $\lambda_{2}\left(t_{j}\right)$ by Lemma 3.9 above. Then, standard arguments as before show that $\varphi_{j}(r) x_{i} / r \rightarrow \varphi(r) x_{i} / r$ where $\varphi$ satisfies (3.10) with $W$ replaced by $t_{0} V$ and $\lambda$ replaced by $\lambda_{2}\left(t_{0}\right)$, i.e. there are $n$ nonradial eigenfunctions corresponding to $\lambda_{2}\left(t_{0}\right)$. Setting $\tilde{V}=t_{0} V$, we see that $\tilde{V}$ is the desired potential.

It remains to prove Lemma 3.9. When the potential $W$ is identically zero, Lemma 3.9 is well known. The arguments used in proving Lemma 3.9 in this special case carry over to the general case. Thus the proof is omitted. Q.E.D.

REMARK. It is easy to prove that for any smooth function $V$, the multiplicity of the second eigenvalue of $\Delta+V$ in a bounded smooth domain $\Omega$ in $\mathbf{R}^{2}$ with zero Dirichlet boundary data is at most three (see e.g. the arguments in [1]). Hence Theorem 3.8 shows that this is in general best possible.

NOTE ADDED IN PROOF. It is also possible to construct a potential $V$ which is a modification of $\alpha /|x|^{2}, 0<\alpha<1$, such that the nodal domain conjecture for (1.1) does not hold. We wish to thank E. N. Dancer for pointing this out to us.

\section{BIBLIOGRAPHY}

1 S.-Y. Cheng, Eigenfunctions and nodal sets, Comment. Math. Helv. 51 (1976), 43-55.

2 R. Courant and D. Hilbert, Methods of mathematical physics, vol. 1, Interscience, New York, 1953.

3 B. Gidas, W.-M. Ni and L. Nirenberg, Symmetry and related properties via the maximum principle, Comm. Math. Phys. 68 (1979), 209-243.

4 C.-S. Lin, On second eigenfunctions of the Laplacian in $\mathbf{R}^{2}$, preprint.

5 W.-M. Ni and R. Nussbaum, Uniqueness and nonuniqueness for positive radial solutions of $\Delta u+f(u, r)=0$, Comm. Pure. Appl. Math. 38 (1985), 67-108.

$6 \mathrm{~W} .-\mathrm{M}$. Ni and J. Serrin, Existence and non-existence theorems for ground states for quasilinear partial differential equations. The analomous case, Proc. Accad. Naz. Lincei 77 (1986), 231-257.

7 L. Payne, On two conjectures in the fixed membrane eigenvalue problem, J. Appl. Math. Phys. (ZAMP) 24 (1973), 720-729.

8 S.-T. Yau, Problem section, Seminar on Differential Geometry (S.-T. Yau, ed.), Ann. of Math. Studies, no. 102, Princeton Univ. Press, Princeton, N. J., 1982, pp. 669-706.

\section{Department of Mathematics, National Taiwan University, Taipei, Taiwan}

SChool of Mathematics, University of Minnesota, Minneapolis, Minnesota 55455 